\title{
LA RECEPCIÓN DEL LYSENKISMO EN MÉXICO
}

\author{
Arturo Argueta Villamar \\ Facultad de Ciencias, UNAM \\ Ricardo Noguera \\ Facultad de Ciencias, UNAM \\ Rosaura Ruiz Gutiérrez
}

Facultad de Ciencias, UNAM

\begin{abstract}
RESUMEN
La historia de la biología en México nos muestra numerosos casos en los cuales se discutió sobre la necesidad de optar por la ciencia aplicada en detrimento de la ciencia pura, sobre las relaciones entre ciencia e ideología y entre la ciencia y el poder, que nos permiten investigar hoy la forma en que se dirimieron estos problemas en el pasado. En este artículo se estudian las concepciones y controversias entre dos prominentes científicos mexicanos, Alfonso L. Herrera e Isaac Ochoterena, que tuvieron un papel relevante en las primeras etapas de la Biología en México, y se revisa la hipótesis de la inconmensurabilidad, con la cual se ha tratado este asunto previamente. Se pone bajo análisis centralmente la recepción del lysenkismo, ocurrida durante la primera mitad del siglo $\mathrm{XX}$, con los propósitos de estudiar el problema, los argumentos, los personajes involucrados, $\mathrm{y}$, sobre todo, los efectos que tuvo tal recepción en el desarrollo de la investigación biológica en México, los cuales se prolongaron hasta casi finales de la década de los años sesentas en la Universidad Nacional Autónoma de México.
\end{abstract}

PALABRAS CLAVE: Lysenko, México, siglo XX, biología.

\section{SUMMARY}

The history of Biology in Mexico shows us several cases, where the discussion on the need to choose the applied science at the expense of pure science, and where the relationships between science and ideology and science and power were analyzed. Today, these cases allow us to research, how these problems were solved in the past. In this paper we study the conceptions and controversies between two prominent Mexican scientists, Alfonso L. Herrera e Isaac Ochoterena (who played an important role during the first stages of Biology in Mexico), reviewing the vastness' hypothesis previously used to study this topic. We focus to analyze the acceptance of Lysenkoism, which occurred during the first half of the 20th Century, with the purposes to study the problems, the arguments, the individuals involved and, above all, the effects of this reception on 
the development of biological research in Mexico, which repercussion went on almost until the end of the $60 \mathrm{~s}$ at the National Autonomous University of Mexico.

KEY WORDS: Lysenko, Mexico, $20^{\text {th }}$ century, biology.

\section{INTRODUCCIÓN}

Los temas que se abordan en este artículo se enmarcan en la historia de la ciencia y particularmente de la biología en nuestro país y tienen como punto de partida la disputa entre Alfonso L. Herrera e Isaac Ochoterena, así como la recepción del evolucionismo darwiniano y del lysenkismo y sus efectos sobre las ideas evolucionistas, la investigación biológica y, en general, el desarrollo de la biología en México.

La importancia de analizar a los personajes y sus ideas, deriva de que vivieron en una época durante la cual se definieron los temas y problemas de la investigación biológica de la primera mitad del siglo XX, y se establecieron algunas de las más importantes instituciones de enseñanza e investigación del México actual.

En un trabajo anterior, Argueta ${ }^{1}$ señaló la necesidad analizar, bajo nuevas hipótesis y nuevos documentos, los argumentos y mecanismos institucionales por medio de los cuales Isaac Ochoterena tuvo las posibilidades de cercenar desde la Universidad Nacional Autónoma de México (UNAM), una sólida introducción del darwinismo en México, en la vertiente de la Síntesis evolutiva, ya que precisamente en los momentos en que la también denominada Teoría sintética, se encontraba en gestación y desarrollo, Ochoterena ocupó la dirección del Instituto de Biología de la UNAM, entre 1929 y 1946, es decir, durante 17 largos años.

\section{DOS PERSONAJES FUNDADORES}

\subsection{Alfonso L. Herrera}

El primer esfuerzo sistemático para introducir el darwinismo en México, lo realizó Alfonso L. Herrera (1868-1942) en la última década del siglo XIX. Enrique Beltrán lo califica de «campeón indiscutible», pues fue un evolucio-

1 Argueta, A. (2003), La recepción e introducción el darwinismo en Bolivia y México, un análisis comparativo, tesis de doctorado, Facultad de Ciencias, UNAM, México, 250 pp. 
nista convencido, plasmogenista, ateo ${ }^{2}$ y masón ${ }^{3}$. Orgullosamente autodeclarado alumno de Alfredo Dugés, Herrera mantuvo con él una importante correspondencia en diferentes etapas de su vida ${ }^{4}$, a través de la cual le consulta a Dugés sobre diversos asuntos específicos (identificación de algún ejemplar, observaciones anatómicas, autores, etc.), o solicita su opinión sobre aspectos de la teoría de la evolución, de la cual argumenta para convencer a su respetado maestro. Roberto Moreno de los Arcos lo ubica como «el darwinista más activo y connotado», cuyo mejor trabajo doctrinal es Recueil des Lois...5, y hace notar que fue redactado como un texto de «catecismo», donde Herrera expone sin poner en duda ninguna afirmación, por lo que opina que es su obra más importante dentro de la larga lista de escritos darwinistas y representa la síntesis del movimiento evolucionista en México $^{6}$.

Para Ruiz, Herrera es «el único que en ese período tiene una concepción generalizadora de lo vivo». Lo describe como el más destacado de los naturalistas mexicanos de fines del siglo XIX y principios del XX, situándolo entre los fundadores de la Biología en nuestro país. Es también el único de los científicos nacionales conscientes del momento que viven las Ciencias Naturales, esto es, el momento de transición entre la Historia Natural y una ciencia con un objeto único: la vida; postula que entonces se deja de describir lo vivo para tratar de explicarlo y que es uno de los pocos biólogos en México, que tienen una concepción filosófica clara y consciente 7 .

Herrera publicó su primer artículo científico, donde expone y discute tesis evolucionistas, en $1890^{8}$, cuando contaba con veintidós años y puede decirse que

2 Beltrán, E. (1942), «Alfonso L. Herrera: un hombre y una época», Revista de la Sociedad Mexicana de Historia Natural, tomo III, nos. 1-4, diciembre, p. 209.

3 Beltrán, E. (1977), Medio siglo de recuerdos de un biólogo mexicano, México, IMERNAR, p. 64.

4 BeltrÁn, E. (1945), «Datos y documentos para la historia de las ciencias naturales en México. II Correspondencia de Alfredo Dugés con Alfonso L. Herrera (1888-1893)», Revista de la Sociedad Mexicana de Historia Natural, tomo VI, nos. 1-2, junio, pp. 99-106.

5 HERRERA, A. L., (1897a). Recueil des Lois de la biologie generale.

6 Moreno de los Arcos, R. (1984), Las polémicas del darwinismo en México, México, UNAM, pp. 37-39.

7 RUIZ, R. (1987), Positivismo y evolución: Introducción del darwinismo en México, México, UNAM, pp. 83 y ss.

8 BeltrÁn, E. (1968), «Alfonso L. Herrera (1868-1942). Primera figura de la Biología mexicana», Revista de la Sociedad Mexicana de Historia Natural, tomo XXIX, diciembre, p. 79. Señala que fue escrito en 1887 , lo que significaría que la primera aportación evolucionista de Herrera fue hecha a los 19 años El dato ha sido verificado y la fecha correcta es la que aporta Moreno. 
entre 1890 y 1904 desarrolló la mayor parte de sus ideas evolucionistas y escribió la casi totalidad de sus artículos relativos a este tema ${ }^{9}$. Posteriormente incluyó el darwinismo en diferentes secciones de cuatro libros, que hacían parte de un conjunto dedicado a la enseñanza y que dio a la imprenta entre 1924 y $1925^{10}$.

Herrera es el introductor del evolucionismo darwiniano en México, pero Ruiz le señala dos grandes carencias: no realizó investigaciones con el propósito específico de comprobar o ampliar alguna de las tesis evolucionistas, probablemente porque consideró al darwinismo una teoría terminada y completa, y mantuvo opiniones ligadas a las tesis de Lamarck y de Haeckel, ya que rechaza la existencia de contradicciones entre las teorías de Lamarck y Darwin, y se plantea el evolucionismo como la suma mecánica de ambas ${ }^{11}$. Como lo ha señalado Ruiz $^{12}$, para Herrera al igual que para muchos evolucionistas de la época, no hay ninguna contradicción significativa, entre Darwin y Lamarck ${ }^{13}$.

Ruiz ha analizado en otro texto ${ }^{14}$ las tesis herrerianas sobre el núcleo duro del evolucionismo: la variación, la selección natural, la lucha por la vida, la adaptación, la herencia, la divergencia de caracteres, el gradualismo y el pro-

9 Una relación, quizá incompleta, de la obra herreriana sobre evolucionismo, en esa década y media, es la siguiente: «Nota acerca de los vertebrados del Valle de México» (1890); «El valle de México considerado como provincia zoológica» (1890); «Nota relativa a las causas que producen atrofia de los pelos. Refutación de un argumento de M. Quatrefages» (1891); «Medios de defensa en los animales» (1892); «Fauna del Lago de Texcoco. Notas acerca de la zoología del Lago de Texcoco y sus alrededores» (1895); «Filosofía comparada. El animal y el salvaje» (1895-1896); «Les Musées de l'avenir» (1895-1896); «La atmósfera de las altitudes y el bienestar del hombre» (1896); «El clima en el Valle de México y la biología de los vertebrados» (1897b); Recueil des lois de la biologie générale (1897); «El ajolote sufre la metamorfosis general en la clase de los batracios, por aumento de nutrición y no por cambio de medio» (1899a); «El origen de los individuos. La construcción de los organismos por las condiciones internas» (1899b); Herrera y Vergara. «La vie sur les hauts plateaux. Influence de la pression barometrique sur la constitution et le développement des êtres organisés...» (1899c) y Nociones de Biología (1904).

10 Se trata de la Botánica (1924a); la Zoología (1924b); la Mineralogía y Geología (1925) y su obra mayor Biología y Plasmogenia (1924c), que es la reelaboración de Nociones de Biología (1904).

11 En la edición de 1924 de Biología y Plasmogenia, y con relación al debate postdarwinista, se inclina a favor de los neo-lamarckianos, en contra de las otras dos tendencias: los darwinistas ortodoxos y los neodarwinistas.

12 RuIZ, R. (1987), Positivismo y evolución: Introducción del darwinismo en México, México, UNAM, p. 83.

13 Herrera, A.L. (1924), Biología y plasmogenia, México, Herrero Hermanos, p. 412.

14 RuIz, R. (1987), Positivismo y evolución: Introducción del darwinismo en México, México, UNAM, pp. 106-137. 
greso, y revisó también el tratamiento que Herrera dio a temas más generales, como los conceptos de especie, de evolución, de unidad de plan, del darwinismo y el neodarwinismo, así como sus ideas y opiniones sobre el papel de la ciencia, entre otros.

Herrera elaboró una versión propia del evolucionismo, que muestra una gran influencia de las ideas de Lamarck, Haeckel y Darwin, en ese orden de importancia; por ejemplo, respecto a la Variación, aceptó como motor de cambio al uso y desuso de los órganos y a la influencia directa del medio y rechaza que tales modificaciones puedan aparecer por azar, por lo que su concepción es absolutamente lamarckiana. Muy ligado a lo anterior, considera que la Adaptación es la facultad de acomodamiento a las diferentes condiciones de vida, es un proceso en el cual el organismo responde con una modificación estructural, funcional o conductual, pues tiene como base la variabilidad de los seres vivos. Por ello mismo, todas las variaciones son o pueden ser adaptativas, aun las que no son, se producen por la interacción ambiental, tales como las monstruosidades y, de hecho, adaptación es sinónima de evolución. En este punto Herrera está muy influenciado por Haeckel, a la mitad entre Lamarck y Darwin.

En la concepción herreriana, la Selección natural es el proceso en el que los seres más aptos en la lucha por la vida tienen mayores posibilidades de persistir, por lo que parece una refinación del concepto de Darwin, pues Herrera subraya «la mayor probabilidad» de persistencia. Tal vez - señala Ruiz - la diferencia se deba a que Herrera está pensando en individuos y Darwin se está refiriendo a variedades y poblaciones. Herrera introduce en este punto un planteamiento importante para no confundir la causa con el efecto, reafirma que la selección natural no es una fuerza creadora ni una entidad inteligente y que no hay que personificarla ni menos deificarla.

En el tema de la Herencia, Herrera retoma las ideas de la herencia como fuerza conservadora y la variación como fuerza de cambio, que es una posición típicamente haeckeliana, y sigue a Lamarck en cuanto a la herencia de las modificaciones adquiridas. Recordemos que este es un punto central en Lamarck y en Haeckel, mientras que Darwin lo acepta pero no le da la importancia que ellos le otorgan. Herrera admite que los cromosomas contienen a los caracteres hereditarios, pero no acepta la tesis weismaniana de la separación entre soma y células germinales, porque el «organismo» es una unidad, porque ni la cromatina puede vivir aislada ni la «evolución verdadera» se da sólo en el plasma germinal, sino en el ser vivo como unidad completa.

Herrera, con todo y su sesgo lamarckiano, es un introductor del darwinismo en la biología, con lo cual establece una ruptura con la historia natural, 
disciplina a la que también contribuyó y enriqueció enormemente. En este sentido es interesante conocer que Herrera planteó, sin embargo, que cada disciplina debía tener su sitio y su espacio, que no debía favorecerse una en lugar de la otra, es decir, que no debíamos «matar a la Historia Natural en nombre de la Biología» ${ }^{15}$.

Debe agregarse que al inicio del siglo, entre 1900 y 1929, Herrera dedicó grandes esfuerzos a la biología aplicada y a la creación y administración de instituciones científicas, así como a la enseñanza en la Escuela Nacional Preparatoria y en la Escuela Normal para Maestros ${ }^{16}$, entre otras, y fue en esa etapa en la que escribió los libros señalados antes, dedicados a la enseñanza media y a la divulgación ${ }^{17}$.

\subsection{Isaac Ochoterena}

Ochoterena (1885-1950) publicó algunos artículos sobre el tema evolucionista, en el Boletín de la Dirección de Estudios Biológicos, seguramente impulsado por su jefe y director Alfonso L. Herrera, el cual puso empeño en que el boletín sirviera de palestra para que, como parte de las actividades teórico-

15 Herrera, A. L. (1924), Biología y Plasmogenia, México, Herrero Hermanos, p. 496.

16 Op. cit. p. 494 (cursivas nuestras). Desde la perspectiva de la enseñanza, afirma que es necesario desarrollar lo mejor de la historia natural para construir a la biología y que ningún maestro de biología puede ser buen maestro y enseñar la teoría de la evolución si no es naturalista. «De la misma manera que para ser ingeniero o abogado se necesita una amplia preparación especial, para ser biólogo es indispensable prepararse en ciencias naturales, estudiar plantas y animales en el laboratorio y en el campo, conocer la geología y la paleontología y, muy especialmente, la química, la física y la físicoquímica. Un profesor o investigador que no posea estos conocimientos jamás podrá entender ni enseñar las teorías e hipótesis de la evolución, basadas en infinidad de hechos y minuciosidades que sólo abarca el naturalista viajero y clasificador y a este respecto se recordará que Darwin, Wallace y Haeckel, antes de publicar sus inmortales obras, hicieron labor amplia y militante de naturalistas».

17 Beltrán, E. (1968), «Alfonso L. Herrera (1868-1942). Primera figura de la Biología mexicana», Revista de la Sociedad Mexicana de Historia Natural, tomo XXIX, diciembre, pp. 76-77. Dice que en carta dirigida a él en febrero de 1930, a propósito de comunicarse los planes mutuos sobre próximas publicaciones, Herrera le dice «He tenido la idea de publicar un periódico exclusivamente dedicado a la teoría de la Evolución... pero no haré nada si usted cree que este periódico pueda perjudicarlo en lo que se refiere a su proyecto de periódico científico de que me habló....». En esos difíciles momentos para los dos, posteriores al desmantelamiento de la Dirección de Estudios Biológicos, ni la proyectada revista de Beltrán, ni los propósitos de Herrera, se pudieron llevaron a cabo. 
prácticas de la institución, diversos colaboradores hicieran difusión de las ideas evolucionistas ${ }^{18}$.

Primero tradujo y publicó en 1916, el artículo de De Vries «Principios de la Teoría de la Mutación», y en el mismo tomo publicó otro al que tituló «Comentarios a la teoría de la mutación», en donde señala que De Vries propone la posibilidad de las variaciones bruscas y sin transición, es decir, a saltos, frente a lo que comenta: «Todas las observaciones de la distribución de plantas y animales y todas las experiencias de la Anatomía experimental, demuestran que con mayor extensión y amplitud que los cambios por salto, debido ya a uno, o lo que es más probable a varios factores mal determinados aún, existen las pequeñas variaciones producidas por las causas lentas que, obrando de acuerdo con el tiempo, tienen su origen en la mutabilidad eterna del medio que rodea a los seres» $\rangle^{19}$, con lo que deja claro que se opone a la variación saltacionista y se adhiere al gradualismo.

De Vries publicó su obra sobre la teoría de las mutaciones de 1901 a 1903. La recepción, traducción e introducción de Ochoterena tiene efecto entre los biólogos mexicanos unos 15 años después, pero nuestro autor lo hace para marcar su diferencia de opinión con el mutacionismo y reafirmar su adhesión a la influencia del medio y al gradualismo que ubica como lamarckiano-darwiniano, de la siguiente forma: «La cuestión de los caracteres adquiridos está, a nuestro juicio fuera de duda, ya que, como dice muy bien M. Edmond Perrier: «Quien dice evolución, dice adquisición de caracteres» ${ }^{20}$, con total resonancia spenceriana, y finalmente subraya «Para terminar este trabajo, manifestaremos que hay otro punto en que los razonamientos del señor profesor De Vries no nos convencen; nos referimos a la exclusión absoluta de las variaciones lentas conforme las suponen y aceptan las teorías de Lamarck y Darwin $\rangle^{21}$.

En la segunda década del siglo XX, el entonces profesor de la Escuela Nacional Preparatoria, Isaac Ochoterena elaboró un libro de texto para las lecciones sobre el tema, en esa institución. Dicho texto, al igual que los de Du-

18 Ver también PÉREZ Amador, M. (1917), «La Unidad Universal»; HerrerA, A. L. (1917), «Discurso de Inauguración»; OCHOTERENA, I. (1916a), «Principios fundamentales de la teoría de la mutación» de Hugo de Vries (traducción), Boletín de la DEB, t. I, pp. 286-296; OCHOterena, I, (1916b) «Comentarios a la teoría de la mutación», Boletín de la DEB, t. I, pp. 295-301; OCHOterenA, I., (1917), «Algunas ideas fundamentales de la obra de Lamarck», Boletín de la DEB, t. II, pp. 180-183; HerRERA, A. L., (1918) «Insectos homocrómicos y miméticos mexicanos», entre otros.

19 OCHOTERENA, I. (1916b), p.301.

20 Ibidem, p. 296.

21 Ibidem, p. 301. 
gés y Herrera, fueron editados expresamente para el nivel de enseñanza media superior y al igual que aquellos, sirvió también de vehículo para discutir las ideas evolucionistas en México.

La redacción del libro, denominado Lecciones de Biología ${ }^{22}$, fue solicitada por el director de la Escuela Nacional Preparatoria, Vicente Lombardo Toledano a Ochoterena, en ese momento profesor y jefe del Departamento de Ciencias Biológicas.

El libro introduce al estudiante, a través de varias lecciones hacia los asuntos de la herencia, el evolucionismo, la teoría de la descendencia, la variación en Lamarck, en Darwin y por salto, así como la vida de Darwin. Al abordar el evolucionismo, plantea las posiciones de los espermatistas y de los ovistas, discute y critica la teoría del soma y las células germinales de Weismann y, al igual que Herrera, afirma que las posiciones de Lamarck y Darwin no son incompatibles y que se puede tomar de cada una lo que tienen de positivo.

Es notable que a casi 20 años de la opinión expresada por Herrera en sus Nociones de Biología (1904), Ochoterena repita el mismo esquema, con lo que definitivamente se ubica también en la perspectiva neolamarckiana, dentro de las corrientes postdarwinianas,

El programa del curso, denominado «Curso sintético de Biología», para el cual fue preparado el libro, contiene, entre otros, los siguientes capítulos: «La herencia, transmisibilidad de los caracteres de la raza y de los individuos. Teorías generales acerca de la herencia. El Mendelismo. Los caracteres adquiridos y el mecanismo de su transmisión hereditaria. Variación darwiniana y sus leyes. Lucha por la existencia y la selección natural. Pruebas anatómicas, paleontológicas y embriológicas del transformismo. Crítica de las teorías evolucionistas $»^{23}$.

Precisamente en la lección XI, dedicada a La Herencia, el autor introduce un subcapitulo al que denomina «Caracteres adquiridos». Dice que ilustres teorizantes como Weismann o Spencer han disertado sobre el tema «demostrando por medio del razonamiento, el primero que no se heredan y el segundo que sí se heredan los caracteres adquiridos ${ }^{24}$. Para Ochoterena esas discusiones están superadas, pertenecen a la historia de la Biología y no reflejan los conceptos actuales que se esclarecen más por observación y experimentación, que por el principio de autoridad.

22 Ochoterena, I. (1922), Lecciones de Biología, México, Secretaría de Educación Pública, $222 \mathrm{pp}$.

23 Ibidem, pp. 5-6 (cursivas nuestras).

24 Ibidem, p. 101. 
Ochoterena defiende la idea de la herencia de caracteres adquiridos en el proceso de adaptación del organismo al medio y remite a los lectores al ejemplo de los tripanosomas resistentes al Atoxyl, medicamento con el cual se les combatía. Señala Ochoterena: «... pero si la dosis empleada, bien sea de Atoxyl puro o combinado con la emetina o con otros medicamentos, es insuficiente y si se suministra con cierta constancia, los protozoarios de que tratamos se acostumbran a la sustancia tóxica y constituyen una raza resistente que conserva este carácter adquirido a través de centenares de generaciones ... $\rangle^{25}$.

Este y otros son los ejemplos que Ochoterena brinda a los estudiantes para demostrar que la observación y la experimentación permiten resolver la controversia, sobre el tema. Después de presentarlos uno a uno, concluye que: «Estos casos tan elocuentes y probatorios, cuyos resultados son concordantes, nos permiten afirmar que, aunque desconocemos exactamente su determinismo, son en gran número de ocasiones, hereditarios los caracteres adquiridos $\rangle^{26}$.

A partir de ambas afirmaciones, podemos señalar que entre 1917 y 1922, no hubo cambios en su información y enfoque sobre este tema crucial. En 1922 Ochoterena cuenta ya con 37 años y sus opiniones sobre el tema, no difieren absolutamente en nada, de las de su maestro Herrera.

Efectivamente, también para Ledesma, ${ }^{27}$ esa etapa académica de Ochoterena es una etapa de coincidencia con los intereses de Herrera y «podría pensarse que aún no ocurría la confrontación entre ambos, más bien había una influencia teórica ...» y agrega que «sería más tarde cuando entablen una competencia académica la cual represa intereses radicalmente distintos y concepciones discrepantes».

Los documentos muestran que Ochoterena incursionó entre 1917 y 1922 en el tema, y como lo ha señalado Meléndez ${ }^{28}$ lo hizo intermitentemente a lo largo de los años posteriores en diversas obras. Le otorgó una gran preponderancia al trabajo descriptivo, que por una parte puede verse como la continuación de una línea de la historia natural y, por la otra, su convicción de indagar en las relaciones que existen entre la forma y la función para precisar las correlaciones entre el individuo y el medio y conocer cómo actúan los excitan-

25 Ibidem, p. 103 (cursivas nuestras).

26 Ibidem, p. 104 (cursivas de Ochoterena).

27 LeDESMA, I. (1998) El conflicto entre Alfonso L. Herrera e Isaac Ochoterena y la institucionalización de la Biología en México, tesis doctorado, Facultad de Ciencias, UNAM, México, p.131.

28 MelÉndeZ, J. L. (1998), Isaac Ochoterena, 1885-1950, México, Gobierno del Estado de Puebla, p. 65. 
tes sobre los seres vivos y cómo éstos responden a los estímulos ${ }^{29}$. Es frecuente ver en los artículos de florística, neurología, fisiología e histología de Ochoterena, la mención a los conceptos de evolución, selección, adaptación y supervivencia, entre otros, pero sin profundizar en ellos.

\subsection{Similitudes conceptuales}

Aunque hay algunas pequeñas diferencias entre las visiones evolucionistas de Herrera y Ochoterena, es claro que comparten ideas similares, por ejemplo, la fuerte convicción por los supuestos de la herencia de caracteres adquiridos, idea que se encuentra en el Darwin de la primera a la sexta edición de El origen de las especies ${ }^{30}$. Aun cuando realmente se dio una ruptura entre Lamarck y Darwin, que consistió principalmente en la inclusión del azar, concepto que rompe con una teoría teleológica e inicia una teoría donde el resultado es contingente, sin embargo, las ideas sobre la herencia de caracteres adquiridos que preconizó Herrera, fueron parte del andamiaje darwiniano en el que también se movió Ochoterena.

En este sentido, Herrera al igual que Ochoterena, creían que la herencia era un proceso dinámico que no debía ser considerado como algo estático y mecanicista, y en donde el medio jugaba un papel fundamental. Convincentemente afirmaba Herrera en forma similar a Darwin que los caracteres adquiridos tienen tanta más seguridad de ser transmitidos cuanto mayor es el tiempo que el organismo ha estado sometido a la acción de las causas modificadoras o las generaciones sucesivas ${ }^{31}$.

Desde 1897 Herrera sostenía que el motor de cambio era el uso y desuso y la influencia directa del medio principalmente en las plantas, una variedad nueva o una nueva especie distinta al original era resultado por el puro efecto del medio ${ }^{32}$. Herrera sostuvo una idea similar en Nociones de Biología y señaló en Biología y Plasmogenia, que: «Todos los seres varían constantemente

29 Ochoterena, I. (1950), Tratado Elemental de Biología, México, Botas, p. 2.

30 Las ideas de la herencia en Darwin se han minimizado a partir de algunos de los errores contenidos en su teoría de la Pangénesis. Sin embargo, sus propuestas sobre la herencia son absolutamente congruentes con sus ideas evolutivas. Es importante tenerlo en cuenta si se quiere hacer una mejor interpretación del darwinismo original y del que en las primeras décadas del siglo XX fue recibido en los diferentes países del globo. Las ideas de Darwin sobre la herencia, son el tema central de la tesis doctoral de Ricardo Noguera.

31 Herrera, A.L. (1924), Biología y plasmogenia, México,Herrero Hermanos, p. 368.

32 Herrera, A. L. (1897a), Recueil des Lois de la biologie generale, p. 74 
bajo la influencia del medio, cambio de condiciones, clima, terreno, alimento, aire, luz altitud, uso y falta de uso. La adaptación exige, de acuerdo a Herrera, que el protoplasma se modifique e incluso que los órganos se transformen profundamente adaptándose a las nuevas necesidades ${ }^{33}$.

Ochoterena aceptó los principios darwinistas de la evolución que operan sobre una variación producida por los cambios ambientales. Las diferencias entre Darwin y Ochoterena residían en que mientras Darwin vacilaba en su consideración sobre si la variación adaptativa era más importante o era la que él llamaba «sport», Ochoterena pensaba que la variación más importante es esta última, a la que llama mutación, aceptando los supuestos de Hugo De Vries. ${ }^{34}$ Adicionalmente, a pesar de que conocía la teoría cromosómica de Morgan, la aceptaba con reservas, pues seguía considerando a finales de los años cuarenta que las alteraciones de los genes o genas (como él los llamaba) estaba aún por confirmarse, ya que estaba convencido de que algunos factores radicaban en el núcleo y otros en las estructuras del protoplasma. Aun más, consideraba que de aceptarse en su forma y en su fondo las ideas de Morgan y su escuela, se llegaba en consecuencia, a la negación total de la evolución orgánica puesto que sólo aparecerían los caracteres existentes, sin posibilidad de adquirir otros nuevos ${ }^{35}$.

3. DISPUTA INSTITUCIONAL DISFRAZADA DE CONTROVERSIA ENTRE CIENCIA PURA Y CIENCIA APLICADA

Cuando Ochoterena llegó al Instituto de Biología, en 1929, sus compañeros de ruta, el Dr. Fernando Ocaranza ${ }^{36}$, M. Moctezuma y S. Morones, que escriben el «Programa de Trabajo del Instituto de Biología», señalaron claramente que «... no se consideraba necesario crear el área de estudios correspondiente a la biología general o dedicar un espacio a los estudios del concepto de la vida y

33 Herrera, A. L. (1924), Biología y plasmogenia, México, Herrero Hermanos, p. 407.

34 Ochoterena, I. (1950), Tratado Elemental de Biología, México, Botas, , p. 262. Debemos recordar que en su texto de 1917 no aceptó los cambios per saltum de De Vries, pues le pareció que iban en contra del gradualismo darwiniano. Ver notas 22 y 24.

35 Ibidem, p. 264.

36 En ese entonces director de la Facultad de Medicina (1924-1934) y posteriormente rector de la UNAM, durante el breve período comprendido entre noviembre de 1934 y septiembre de 1935. 
del origen de la misma», bajo el argumento de que lo que «requería el país eran productos de la ciencia aplicada y no de la biología teórica» ${ }^{37}$.

Es importante recordar que la institución precursora que sirvió de base al establecimiento del Instituto de Biología, fue la Dirección de Estudios Biológicos (DEB), se creó justamente bajo la perspectiva de «tomar a su cargo, tanto en el campo como en el laboratorio, el estudio y la investigación de la flora y la fauna, para conocerlas en su conjunto y en sus detalles, para clasificar sus especies y para aprovechar sus productos, en beneficio de la Nación y de sus habitantes» ${ }^{38}$, por lo que tuvo una orientación tanto teórica como fuertemente aplicada, alcanzando varios éxitos, por ejemplo, en el levantamiento de los inventarios y mapas florísticos y faunísticos estatales ${ }^{39}$, el acrecentamiento del Herbario Nacional y en el combate a plagas agrícolas, como la de la langosta ${ }^{40}$, entre otras muchas actividades de difusión y comunicación de las ciencias biológicas.

El párrafo sobre el nuevo perfil institucional y las áreas que tendría el futuro instituto, se elaboraron para demarcarse absolutamente de los trabajos de Herrera, quien en la Dirección de Estudios Biológicos, además del cargo de Director, trabajaba justamente en la Sección de Biología, a cargo del Dr. Emiliano Torres ${ }^{41}$, pero al mismo tiempo significó que en el centro de investigaciones biológicas más importante del país, en esos momentos, se produjera una bajísima permeabilidad y rechazo a estos temas y no se produjeran avances en dos de las cuestiones centrales de la biología del siglo XX: el origen de la vida y el evolucionismo.

El Instituto de Biología de la UNAM (IBUNAM), con propósitos meramente aplicados, se estableció para sustituir a la DEB, cuyo sentido fundamental de investigación fue la ciencia aplicada. Es decir, que para los tomadores de decisiones de la época, no era lo mismo la ciencia aplicada de la DEB, que la ciencia aplicada del IBUNAM, lo cual por supuesto es un absurdo total, que nos muestra que el propósito fue más una disputa por el control de las instituciones, que un cambio en los enfoques y las políticas de investigación.

37 Ocaranza, F.; Moctezuma, M.; Morones, S. (1929), Programa de Trabajo del Instituto de Biología, Archivo del Instituto de Biología, CESU, UNAM, p. 1 (cursivas nuestras).

38 RouAix, P. (1942), «El profesor Alfonso L. Herrera y su labor en la Comisión de Parasitología Agrícola», Rev. de la Soc. Mex. de Hist. Nat., 4, pp. 193-199.

${ }^{39}$ Beltrán, E. (1953), «Hechos salientes de la biología mexicana en el siglo XX», Memoria del Congreso Científico Mexicano, t. VII, p. 463.

40 BeltrÁn, E. (1977), Medio siglo de recuerdos de un biólogo mexicano, p. 45.

41 Ibidem, p. 26. 
Recordemos que si bien Herrera tenía como propósito importante la investigación teórica, también fue un gran cultivador de la investigación aplicada e incluso del diseño, creación y administración de instituciones científicas de carácter práctico o aplicado, como fueron los programas de trabajo de dos de las instituciones científicas más importantes de México al inicio del siglo XX: la Comisión de Parasitología Agrícola (1900-1907) y la Dirección de Estudios Biológicos (1915-1929). Ese interés por la investigación aplicada es propio de un investigador de la historia natural (y no sólo la elaboración de inventarios y listados) y en este caso, Beltrán anota que Herrera incluso le apoyó con algunas ideas y propuestas de aplicación, durante la fase de proyecto del que después sería el Instituto Biotécnico, que funcionó bajo la dirección de Beltrán entre 1934 y $1940^{42}$.

El desenlace de la disputa institucional significó para Herrera el eclipse dentro de las instituciones públicas del país, mientras que para Ochoterena fue el paso para su afianzamiento en el puesto de dirección del Instituto de Biología, durante más de quince años, pero al mismo tiempo significó que en lo personal no profundizara en los estudios de lo que se denominó «biología general y origen de la vida», con lo cual personalmente siguió siendo lamarckista, como ya lo era entre 1917 y 1922 pero, desafortunadamente, hubo otros resultados aún más negativos.

\section{SOBRE LA HIPÓTESIS DE LA INCONMENSURABILIDAD RESPECTO AL EVOLU- CIONISMO}

Para Ruiz $^{43}$ es claro que Herrera comparte con Lamarck, la artificialidad de las clasificaciones; las especies no son todas contemporáneas sino que descienden unas de otras; la variación es producto de los cambios en el medio ambiente; las condiciones de vida influyen en la organización y forma general de los animales (uso y desuso); tendencia al aumento de complejidad; gradualismo; principio de igualdad entre fenómenos físicos y biológicos; generación espontánea en plantas y animales sencillos; la idea de serie, pero en la cual Lamarck excluye a los seres no vivos y Herrera los incluye. Herrera sigue a Haeckel en el tema de la oposición entre herencia y adaptación, mientras que para Darwin la oposición se verifica entre la herencia y la variación y no toda

42 Ibidem, pp. 127-128.

43 RuIZ, R. (1987), Positivismo y evolución: Introducción del darwinismo en México, México, UNAM, p. 127. 
variación es adaptativa. Con relación al gradualismo, se trata de un tema común entre Lamarck, Darwin y Herrera, pero debe agregarse que Herrera conoció la propuesta mutacionista de De Vries y consideró que algunos cambios súbitos y bruscos podrían deberse a las mutaciones.

Por su parte, Ochoterena dedicó algunas reflexiones a la selección natural y al origen de las especies, la adaptación, la supervivencia y otros temas similares, de manera intermitente a lo largo de los años posteriores en diversos textos ${ }^{44}$, pero no cabe duda que al no existir el espacio institucional para hacerlo ampliamente, intercambiar y discutir con los colegas, no hizo sino repetirse hasta adquirir rasgos de circularidad, sin diálogo con las corrientes contemporáneas.

$\mathrm{Su}$ obra principal, respecto a este tema, siguió siendo las «Lecciones de Biología» de 1922, que se convirtieron en el «Tratado Elemental de Biología» a partir de la segunda edición de 1929 y que continuó reimprimiéndose muchos años después ${ }^{45}$. En la edición de 1950, que estaba reelaborando en el momento de su muerte ${ }^{46}$, en el capitulo XV denominado «Teoría para explicar la herencia. Teoría cromosómica. Crítica y estado actual», Ochoterena señala que Mendel y Morgan admiten las variaciones denominadas por ellos mutaciones, pero que afirman que no pueden ser previstas y que carecen de valor como agentes modificadores de la herencia ${ }^{47}$.

Reitera que de aceptarse las ideas de los genetistas, se niega totalmente a la evolución orgánica, ya que solamente aparecerían en los nuevos individuos los caracteres de sus antepasados, sin posibilidad de adquirir otros nuevos y, «si apareciera algún carácter nuevo, se perdería, ya que vendría a formar par-

44 Ver «Algunos conceptos fundamentales acerca de la evolución de los seres vivos» (1931); "Algunas orientaciones fundamentales de la biología» (1940); Estudio biológico de los órganos de los sentidos (1941); entre otros.

45 De lo revisado a la fecha, las ediciones y reimpresiones fueron las siguientes: OCHOTERENA, I. Lecciones de Biología, 1ª. Ed., SEP, 1922; Tratado Elemental de Biología, 2a. Ed., Imprenta Murguía, 1929; 3ª . Ed., Imprenta Murguía, 1936; 4a. Ed., Imprenta Universitaria, UNAM, 1937; 5a . Ed., Editorial Botas, 1940; 7ª Ed., Editorial Botas, 1944; ${ }^{\text {a }}$. Ed., Editorial Botas, 1946 (en vida); 9 . Ed., Editorial Botas, 1950 (póstuma); 11 a . Ed., Editorial Botas, 1960; $13^{a}$. Ed., Editorial Botas, 1970, 493 pp. Es claro que el libro se utilizó ampliamente para dar las lecciones de Biología a los estudiantes de la Escuela Nacional Preparatoria por casi cinco décadas, entre 1922 y 1970, y podemos suponer el enorme impacto de esta obra, por la cantidad de años que estuvo en circulación entre numerosas generaciones de estudiantes de México.

46 MARTín DEL CAMPO, R. (1950), p. XI; dice en la nota introductoria que ayudó a Ochoterena a redactar, a partir de los apuntes de éste, el apartado final del capítulo XV: «Teoría para explicar la herencia. Teoría cromosómica. Crítica y estado actual».

47 Ibidem, p. 255 
te, exclusivamente, del llamado plasma somático, sin afectar el plasma germinativo, único que, según ellos, puede transmitir hereditariamente los caracteres». Frente a esto afirma que: «La experiencia, sin embargo, demuestra la existencia de cambios en los seres vivos, cambios transmisibles a la descendencia .... ${ }^{48}$. Y unos párrafos después, cierra el tema con la siguiente sentencia: «Esto vuelve a dar actualidad viva y potente a las ideas de Lamarck, uno de los más profundos filósofos de la Biología, quien admitió la persistencia hereditaria de los caracteres adquiridos bajo la influencia del ambiente, caracteres que se conservarán en tanto el individuo no sea sometido a condiciones diversas ${ }^{49}$.

Con base en los hechos y documentos anteriormente citados, en los cuales se muestra claramente que sigue hablando de herencia de caracteres adquiridos en 1950, casi en el mismo sentido que lo hacía en 1922, podemos afirmar que Ochoterena no se diferenció de Herrera superando el darwinismo influido de lamarckismo de aquel, con un darwinismo de corte más contemporáneo, como lo fue el fisherismo entre 1918 y $1933^{50}$.

Por lo expuesto anteriormente, consideramos que la propuesta de Ledes$\mathrm{ma}^{51}$ respecto a la posible inconmensurabilidad entre las posiciones de ambos autores, es inaplicable, ya que en cuanto al tema evolucionista, opiniones polares o discrepancias absolutas nunca existieron, y no las hubo porque las concepciones evolucionistas de Ochoterena de su etapa madura no fueron teóricamente distintas de las que expresó en los años veinte, cuando se formó al lado de Herrera.

Pérez Ransanz nos recuerda que el término fue llevado por Kuhn de la «inconmensurabilidad matemática», entendida como la falta de una unidad común de medida, hacia la filosofía de la ciencia para utilizarla metafóricamente en el terreno de las teorías empíricas para indicar la falta de un lenguaje común que permita su traducción, sin pérdidas ni residuos ${ }^{52}$.

\footnotetext{
48 Ibidem, p.256.

49 OChOterena, I. (1950), Tratado Elemental de Biología, p. 255.

50 MAYR, E. (1992), Una larga controversia. Darwin y el darwinismo, Barcelona, Crítica, p. 155

51 Ledesma, I. (1998), El conflicto entre Alfonso L. Herrera e Isaac Ochoterena y la institucionalización de la Biología en México, tesis doctorado, Facultad de Ciencias México, UNAM, p. 216 y ss.

52 PÉReZ RANSANZ, A. R. (1997), «Cambio científico e inconmensurabilidad», en: VELASCO GómEZ, A. Racionalidad y cambio científico, México, PAIDOS-UNAM, p. 78 (Cursivas de la autora).
} 
Kuhn no analizó, por ejemplo, los posibles casos de «subteorías rivales», aquellas que en el ámbito de un mismo paradigma suponen las mismas leyes o postulados fundamentales, pero que rivalizan con respecto a ciertas hipótesis y que generan ciertas controversias, que se ha desarrollado posteriormente como lo señala Pérez Ransanz, pero tampoco es el caso protagonizado entre Ochoterena y Herrera, ya que entre ellos no hubo teorías ni subteorías rivales, tampoco intraducibilidad, en suma la hipótesis de la inconmensurabilidad entre las teorías que ambos preconizaban, nos parece que es insostenible.

\section{LA RECEPCIÓN DEL MICHURINISMO - LYSENKISMO EN MÉXICO}

Un análisis más completo sobre las concepciones teóricas, las posiciones académicas y las prácticas institucionales de Ochoterena no se agota en lo relativo a su triunfo institucional sobre Herrera o la no inconmensurabilidad entre las perspectivas teóricas de ambos autores, sino que debe ahondar en las ideas en que incursionó posteriormente.

Entre 1946 y 1950, es decir, a finales de la primera mitad del siglo XX, los textos de la octava y la novena ediciones del Tratado Elemental de Biología son claves para entender ese siguiente período, en donde Ochoterena señala los avances del darwinismo, el neodarwinismo de Weismann, el mendelismo, y el mutacionismo de Morgan, Bridge y Sturtevant, entre otros autores, pero nunca menciona a los principales autores evolucionistas de ese momento como Dobzhansky ${ }^{53}$, Mayr ${ }^{54}$, Huxley ${ }^{55}$ y Simpson ${ }^{56}$, Rensch y otros que habían elaborado, desde finales de los años 30, los principales textos y las ideas centrales de lo que entre 1936 y 1947 fue precisamente la vigorosa Síntesis evolutiva o también conocida como Teoría sintética ${ }^{57}$.

Por el contrario, no sólo no recepciona la Teoría sintética, sino que recibe y divulga el michurinismo-lysenkysmo. Ochoterena explica que: «Los agrónomos y biólogos soviéticos han obtenido asombrosos resultados en los últimos

53 Dobzhansky, Th. (1937), Genetics and the origin of the Species, Nueva York, Columbia University Press.

54 MaYr, E. (1942), Systematics and the Origin of the Species, Nueva York, Columbia University Press.

55 HuXLeY, J. (1942), Evolution: the Modern Synthesis, Allen and Unwin, London.

56 SimPSON, G. G. (1944), Tempo and Mode of Evolution, Nueva York Columbia University Press.

57 MAYR, E. (1992), Una larga controversia: Darwin y el darwinismo, Barcelona, Crítica, p. 145 . 
años (...). Han llegado incluso a la obtención de especies nuevas hibridando las existentes por el procedimiento del injerto de plantas pertenecientes no sólo a diversas especies, sino a diversos géneros $\rangle^{58}$.

Es evidente que argumenta sobre la misma base ideológica de lo que se denominó «el darwinismo creador soviético», que se propuso combatir el socialdarwinismo del darwinismo, ya que afirmaba: «De una innegable trascendencia social son estas adquisiciones científicas, pues tienden a invalidar, en lo que a la humanidad se refiere, las conocidas ideas de Malthus, sobre las cuales Darwin basó su teoría de la lucha por la existencia, puesto que aumentan y mejoran la subsistencia del hombre» ${ }^{59}$.

La serie de conferencias que ofreció en 1949 en El Colegio Nacional, dedicadas a la herencia y la evolución, presentan un diseño similar, de las cuales solamente extraemos los títulos de las dedicadas al evolucionismo, que dan prueba de que el tema le fue muy significativo: «Los sistemas evolucionistas: espermatistas y ovistas. Buffón y sus moléculas orgánicas. Erasmo Darwin. La pangénesis de Darwin. Breve exposición de las teorías micromeristas: de Weismann a la genética. El lamarckismo. Las teorías organicistas: Roux y Hertwig. Doctrina de las causas actuales. Ives Delage. Mariano de Lagasca. Lysenko y Michurin. Infecundidad de las teorías micromeristas y su desacuerdo con la Doctrina de la Evolución. Las causas actuales; hechos embriológicos e histológicos. Los trabajos de Lysenko y Michurin y su significación para el progreso de la agricultura y para el bienestar de los pueblos. Resumen y conclusiones» ${ }^{60}$. Estas fueron quizá las 11 últimas conferencias de su vida, dice Ledesma ${ }^{61}$.

Una muestra de la importancia de la labor de recepción y divulgación que hizo Ochoterena, nos la ofrecen sus contemporáneos Alfredo Barrera, Narciso

58 OCHOterenA, I. (1950), Tratado Elemental de Biología, p. 256 (cursivas nuestras).

59 Ibidem, p. 257. Con frecuencia se concluye que la biología rusa de la segunda mitad del siglo XIX y, por extensión las tendencia posteriores, como el michurinismo-lysenkismo, estuvieron en extremo ideologizadas y politizadas, lo cual es cierto en parte, pero frecuentemente olvidamos que en occidente, al mismo tiempo, florecieron las corrientes geneticistas que impulsaron los grandes programas eugenésicos, no menos ideologizados que aquellos . Ver SuÁREZ y LóPez GuAzo, L. (1999), Eugenesia y determinismo biológico en México,tesis de doctorado. Facultad de Ciencias, México, UNAM, p.12

60 El Colegio Nacional. (1969), «Trabajos realizados por El Colegio Nacional durante el año de 1949», Memoria de El Colegio Nacional, IV (4), p. 106 (cursivas nuestras).

61 LEDESMA, I. (2000). «Ochoterena: el hombre, la ciencia y las instituciones científicas», en: OchoterenA, I. (2000), Obra Cientifica, Obras I (selección y comentarios de Ismael Ledesma Mateos y Antonio Lazcano-Araujo), México, El Colegio Nacional, p. 26 
Bassols Batalla y Rafael Martín del Campo cuando escriben, en el prólogo a la edición en español del libro de Morton, La Genética en la URSS, ${ }^{62}$ traducida por ellos mismos, que «fue Ochoterena el primero en nuestro país en percibir la trascendencia de las investigaciones de los biólogos soviéticos en el campo de la Genética». Hacen referencia al ciclo de conferencias de 1949, en donde Ochoterena estimó como «notables e importantísimas las experiencias ideadas y realizadas por los investigadores de la Unión Soviética», de las que pensaba incluir en su Tratado Elemental de Biología, «una noticia especial sobre el tema», pero que le fue imposible ${ }^{63}$.

Con base en los documentos anteriormente citados, está claro que Ochoterena realizó la recepción del michurinismo-lysenkismo, es decir un tipo de lamarckismo, aun cuando los científicos soviéticos se consideraban fieles continuadores de Darwin, en sentido opuesto a los esfuerzos neodarwinistas y de gestación de la teoría de la «Síntesis evolutiva», según la formulación de Huxley ${ }^{64}$, que profundizó la tarea darwiniana comenzada menos de un siglo antes.

Ledesma dice «Qué lejos estaba don Isaac de imaginar las funestas consecuencias de la genética de Lysenko en la URSS, sin embargo, dicho texto nos ayuda a pensar en su interés por estar al tanto de una postura aparentemente innovadora en el campo de la ciencia, y al mismo tiempo de la distancia que en el transcurso de los años estableció con respecto a los temas de biología general, del evolucionismo darwiniano y la genética mendeliana; debido a su plena dedicación al campo de la morfología, y en especial de la histología, de la sensoriedad y de la oncocercosis, a los cuales consagró sus últimos días» ${ }^{65}$.

Este autor le concede a Ochoterena el beneficio de la duda. Nuestra apreciación es que ese beneficio no concuerda con la época (pues algunas de las peores consecuencias ya había ocurrido precisamente una década antes), ni con la información que alguien bien enterado, como Ochoterena, ya sabía a esas alturas del partido, por ejemplo que Trofim D. Lysenko se impuso por la fuerza de la política a Nikolaï Ivanovitch Vavilov, quien era el director del

62 Barrera, A.; Bassols Batalla, N. y Martín Del CAMPo, R.. «Prólogo», en: MorTON, A. G (1953), La Genética en la URSS (trads. Barrera, Bassols y del Campo), México, Ediciones del Índice, p. 5.

63 Martín del Campo colaboró con Ochoterena en la adición de nuevos textos a la edición de 1950. Ver nota 23.

64 HuXley, J. (1942). La evolución. Síntesis moderna, Buenos Aires, Losada, p. 11 y MAYR, E. (1992), p. 144.

65 LedeSMA, I. (1998), El conflicto entre Alfonso L. Herrera e Isaac Ochoterena y la institucionalización de la Biología en México, tesis doctorado, Facultad de Ciencias, UNAM, México, p.152. 
Instituto Pansoviético de Genética Vegetal (o Instituto de Plantas Cultivadas) y una gran celebridad de la genética de la ex - URSS $^{66}$.

Vavilov estuvo en dos ocasiones en México realizando colectas, primero en 1930 y después en 1932, seguramente entró en contacto con Maximino Martínez del IBUNAM al cual cita en el célebre trabajo sobre México y Centroamérica ${ }^{67}$. Unos años antes Bukasov había estado también en México, colectando con los mismos propósitos, bajo las ordenes de Vavilov, por parte del Instituto de Plantas Cultivadas. En 1932 nuestro autor había publicado en Nature el artículo denominado «El papel de Darwin en el desarrollo de las ciencias biológicas» y un año después fue nombrado Vicepresidente del VI Congreso Internacional de Genética. Entre 1935 y 1936, y previendo la posible realización del Congreso Internacional de Genética en Moscú, se propone difundir la obra de Darwin, de T. H. Morgan y de H. J. Müller en la URSS, para lo cual prepara tres volúmenes de las obras escogidas de tales autores y los edita en Moscú y Leningrado en 193768 .

Medawar y Medawar69 nos recuerdan que el mismo año de 1937, se publicó el libro de Dobzhansky ${ }^{70}$ y se suponía que sus aportaciones se discutirían precisamente en el VII Congreso Internacional de Genética que se llevaría a cabo en Moscú, ese mismo año, el cual fue pospuesto dos veces y finalmente se cambió de sede, para llevarse a cabo en Edimburgo, Inglaterra, en 1939. Subrayan que «de hecho se le había elegido a Vavilov como Presidente del Congreso», tanto del de 1937 como del de 1939, pero el gobierno soviético no le permitió asistir a Edimburgo. A cambio de ello, en agosto de 1940, Vavilov

66 VAVILOV, entre otras actividades, continuó la línea de investigaciones que inauguró De Candolle (1820), autor muy citado por Darwin en «El origen....», con referencia a los centros de origen de las plantas cultivadas. Vavilov desarrolló un ambicioso programa de colecta de plantas cultivadas, recorriendo él y sus colegas prácticamente todo el mundo, entre 1916 y 1935 . Véase VAVILOV, N. (1951), Estudio sobre el origen de las plantas cultivadas, Buenos Aires, ACME. Versión en inglés: VAVILOV, N. (1992), Origin and geography of cultivated plants, Cambridge, Cambridge University Press; y VAVILOV, N. (1951), The Origin, Variation, Inmunity and Breeding of Cultivated Plants, Waltham, Mass. J., Chronica Botanica.

67 Vavilov, I. (1931), "Mexico and Central America as the principal centre of origin of cultivated plants of the new World», p. 186. Dice Vavilov que la región es, sin lugar a dudas, uno de los siete centros mundiales de origen de plantas cultivadas.

68 BABKov, V. (1996), «Vavilov, Nikolaj Ivanovich», en: TORT, P. (dir.), Dictionnaire $d u$ darwinisme et de l'évolution, París, PUF, 3 vols. , pp. 4427-4436.

69 Medawar, P. B. y Medawar, J. S.(1988), De Aristóteles a Zoológicos, México, Fondo de Cultura Económica, pp. 187-190

70 Dobzhansky, Th. (1937), Genetics and the origin of the Species, Nueva York, Columbia University Press. 
fue arrestado por la NVDK, se le sometió a un juicio viciado y se le encarceló en Saratov ${ }^{71}$, Ukrania, en donde murió tres años después.

Vavilov fue eclipsado de la discusión sobre la genética vegetal y eliminado de la disputa entre la genética mendeliana y la genética lysenkista-michurinista. El fondo del debate para Lysenko era la prevalencia de la ciencia soviética sobre la ciencia occidental, y también del darwinismo en manos de la ciencia proletaria o de la ciencia burguesa. Con base en la idea del «darwinismo creador soviético», denunció a la genética que preconizaba Vavilov «como una ciencia capitalista que perpetuaba la noción de que hay diferencias cualitativas - afirmando que tenían su origen en los genes - entre plantas, animales o gente. Tales diferencias inmutables no existen, de acuerdo con Lysenko; las diferencias entre los individuos se deben a los efectos del ambiente y pueden ser radicalmente modificadas exponiendo los organismos a retos ambientales apropiados $\gg{ }^{72}$.

Otro grupo de científicos soviéticos había trabajado sobre la base de conceptos como el de Reaktionsnorm, propuesto por el zoólogo alemán Woltereck en 1908. Se consideraba «norma de reacción» la capacidad de un genotipo para expresar distintos fenotipos, de acuerdo a las condiciones ambientales. Bajo esta interpretación, el concepto de genotipo resultaba menos determinista ${ }^{73}$. Efectivamente, Woltereck quería contrarrestar con ello la perspectiva establecida por Johannsen sobre la diferencia entre fenotipo y genotipo, lo que al mismo tiempo significó una reivindicación de la herencia de caracteres adquiridos.

El concepto de Norma de reacción fue retomado en la Unión Soviética por Iván I. Schmalhausen ${ }^{74}$ y reelaborado con las ideas darwinistas que daban preponderancia a la variación adaptativa en el esquema evolutivo, convirtiendo a la Norma de reacción en un concepto central. A partir de ello, los genetistas soviéticos distinguieron entre norma de reacción adaptativa y norma de

71 Saratov, capital de Saratov Oblast, es una ciudad-puerto del río Volga, al sur de Rusia.

72 RuIz, R.; y Ayala, F. J. (1998), El método en las ciencias. Epistemología y darwinismo, México, Fondo de Cultura Económica, pp. 30-31.

73 En Occidente se aceptó totalmente la distinción entre fenotipo y genotipo y posteriormente se consolidó una tendencia que enfatizaba la constancia y eficacia causal del genotipo (una visión genocéntrica) soslayando la complejidad de las interacciones que la Norma de reacción defendía, pero que en Occidente fue desconocida e ignorada hasta alrededor de 1950. Ver SARKAR, S. (1999), «From the Reaktionsnorm to the adaptive norm: The norm of reaction, 1909-1960», pp. 237-238.

74 Ivan Ivanovitch Schmalhausen, fue un zoólogo ruso que se desempeñó como director del Instituto de Morfología y Evolución, entre 1935 y 1948, y como profesor de darwinismo en la Universidad de Moscú, entre 1939 y 1948. 
reacción no adaptativa y crearon un modelo de evolución orgánica, basado en varios principios, entre los que resaltaba la preponderancia del ambiente ${ }^{75}$.

Ante ambos y otros muchos, Lysenko ganó la partida interna, es decir, todos los oponentes de la talla de Vavilov corrieron la misma suerte de aquel y en el mejor de los casos, fueron despedidos de sus trabajos en la enseñanza y la investigación, e incluso retirados de las universidades y sus libros de las bibliotecas, como fue el caso de Schmalhausen a partir de $1948^{76}$.

El gran momento de gloria de la ciencia lysenkista ${ }^{77}$, fue el Congreso de la Academia de Ciencias de la URSS, realizado en 1948 y presidido por V. I. Stalin, un año antes de las conferencias de Ochoterena en El Colegio Nacional. En tal Congreso se abordaron temas absolutamente cruciales y hubo discusiones muy importantes, pero debe señalarse que el procedimiento fue el de votar las resoluciones, sin dejar el tema a la reflexión, a la investigación y al análisis posterior.

La influencia de la corriente lysenkista-michurinista se prolongó en la ex URSS durante más de 25 años, entre 1937 y 1964. Lysenko fue destituido en octubre de 1964, al igual que fueron erradicadas buena parte de las herencias estalinistas. La rehabilitación y las reediciones de la obra de Vavilov habían comenzado desde 1957, aunque un largo tiempo se guardó silencio sobre su arresto y su muerte ${ }^{78}$. Lecourt ha señalado que Vavilov es el Galileo del siglo $\mathrm{XX}^{79}$ y la UNESCO declaró a 1987 como el Año del Centenario de N. I. Vavilov.

75 Schmalhausen expuso y defendió estas ideas en su ponencia en las reuniones que se realizaron para analizar la situación de las ciencias biológicas en la URSS. Actas de la Academia Lenin de Ciencias Agrícolas de la URSS (1949), «La situación de las ciencias biológicas», Editorial en Lenguas Extranjeras, Moscú, pp. 458-464.

76 BABKov, V. (1996), «Darwinisme Russe», en TORT, P. (dir.) Dictionnaire du darwinisme et de l'évolution, París, PUF, 3 vols., p. 1097. Véanse también los calificativos de «reaccionario, anticientífico y morganiano» que Lysenko coloca a Schmalhausen, en el famoso «Informe del Académico T. D. Lysenko acerca de la situación de las ciencias biológicas» de 1948.

77 Algunos autores discuten si el intento lysenkista fue seudociencia o ciencia errónea. ver Rostand, J. (1971), Ciencia falsa y falsas ciencias, Barcelona, Salvat-Alianza, pp. 39-46 y Medawar, P. B.; Medawar. J. S. (1988), P.189.

78 BABKov, V. (1996), «Vavilov, Nikolaj Ivanovich», en TORT, P. (dir.) Dictionnaire du darwinisme et de l'évolution, París, PUF, 3 vols. , pp. 4435-4436.

79 LeCourT, D. (1974), «Introducción», en: El caso Lysenko, Barcelona, Cuadernos Anagrama, p. 13. 


\section{Ciencia y política en el deSARRollo de la Biología en MÉXiCo}

El análisis del desarrollo de las ideas evolucionistas en México, en la primera mitad del siglo XX, nos ha mostrado que en el ámbito del IBUNAM y de El Colegio Nacional, Ochoterena optó por la recepción del lysenkismo, posponiendo la recepción de la teoría sintética. Dicha decisión, realizada más con base en argumentos políticos que científicos, se erigió en un obstáculo epistemológico ${ }^{80}$ para la recepción de otras teorías, en el seno de una comunidad académica. Este obstáculo se mantuvo férreo e inamovible durante varias décadas, en esa comunidad académica, al final de las cuales algunos autores comenzaron a escribir artículos sobre evolucionismo contemporáneo ${ }^{81}$.

Lo que aquí importa reflexionar es que la adopción de un programa de investigación regresivo, por parte de un científico, es un asunto que ocurre todos los días y tiene consecuencias acotadas en la mayoría de los casos al interior de su obra personal o de grupo. ${ }^{82}$ De lo que estamos hablando es de cuando el director o coordinador de un instituto de investigación científica, por una decisión política o burocrática, puede contener el avance de la investigación hacia líneas más progresivas que las que él mismo preconiza.

Los autores que han investigado a Herrera o a Ochoterena a través del estudio general de su obra, como Beltrán ${ }^{83}$ y Meléndez ${ }^{84}$ no han reparado en este hecho, definitivamente de gran importancia para la biología nacional, ya que han orientado sus análisis hacia la tremenda disputa personal e institucional protagonizada entre ambos y la han calificado como un problema personal

80 Bachelard, G. (1987), La formación del espiritu científico, México, Siglo XXI, p. 15.

81 PIÑERO, D. (1996) «La teoría de la evolución en la biología mexicana: una hipótesis nula», Ciencias, no. 42, abril-junio, p. 7. Agrega que después del largo período de Ochoterena, lo sucedió en la dirección Roberto Llamas, que ocupó el cargo por otros largos 22 años, y «quien tampoco dirigió la investigación hacia aspectos evolutivos».

82 Ver LAKATOS, I. (1983), La metodología de los programas de investigación científica, Madrid, Alianza Editorial, pp. 144-146. Señala que abrazar un programa regresivo no es necesariamente signo de obscurantismo, sino que nuevas investigaciones pueden convertir un programa regresivo en uno progresivo.

83 BeltrÁn, E. (1942), "Alfonso L. Herrera: un hombre y una época», Revista de la Sociedad Mexicana de Historia Natural, tomo III, nos. 1-4, diciembre, pp. 201-210.; BELTRÁN, E. (1968), «Alfonso L. Herrera (1868-1942). Primera figura de la Biología mexicana, Revista de la Sociedad Mexicana de Historia Natural, tomo XXIX, diciembre, pp. 37-91; BeltrÁN, E. (1977), Medio siglo de recuerdos de un biólogo mexicano, pp. 21-61.

84 MelÉndez, J. L. (1998), Isaac Ochoterena, 1885-1950, México, Gobierno del Estado de Puebla, 142 pp. 
que derivó en académico. Ledesma señala solamente que en el IBUNAM hubo una marginación de Herrera en la enseñanza, impulsada por Ochoterena, quien preconizó una biología aplicada y abandonó el evolucionismo ${ }^{85}$.

Piñero ha propuesto una explicación sobre la poca o ninguna importancia de la teoría evolutiva en las investigaciones realizadas en el Instituto de Biología durante más de cuatro décadas y encuentra la causa en la preponderancia de la Historia Natural sobre la Biología ${ }^{86}$, favoreciendo la catalogación de los recursos naturales sobre cualquier otra línea de investigación. Efectivamente, el Programa de trabajo de 1929 del IBUNAM fue absolutamente regresivo, pero no hacia la Historia Natural, sino a unos cuantos campos temáticos de la historia natural, pero no es la explicación completa.

En este punto es necesario reflexionar algunas de las paradojas de esta historia, tanto de la historia natural como de la biología en México. En la afirmación de Piñero se hace unívoca la relación entre historia natural y catalogación de recursos, ¿cómo explicarse entonces que en la Dirección de Estudios Biológicos, precedente del IBUNAM, planeada y organizada por historiadores naturales se estudiara y difundiera el evolucionismo?. ¿Cómo explicarnos que en el IBUNAM, en esos momentos el espacio predilecto de la «profesionalización» de la biología, ya que estaba integrado en su mayoría por «biólogos profesionales», no se hayan planteado un programa de trabajo que incluyera los temas cruciales de la biología del siglo XX, tales como el evolucionismo, el origen de la vida o la herencia a través del estudio de la genética? ${ }^{87}$.

Estimamos que debe profundizarse en este tema a través del estudio de los períodos de transición en los procesos de la profesionalización, que no se expresan necesariamente a través del establecimiento de nuevas instituciones, ni mucho menos con el relevo de personas, sino sobre todo en el cambio de paradigmas, conceptos y contenidos teóricos. La ruptura entre el IBUNAM y la DEB, bajo nuestro análisis, fue sólo de personas y de títulos profesionales.

85 Ledesma, I. (1998), El conflicto entre Alfonso L. Herrera e Isaac Ochoterena y la institucionalización de la Biología en México, tesis doctorado, Facultad de Ciencias, México, UNAM, p. 235.

86 PIÑERO, D. (1996), «La teoría de la evolución en la biología mexicana: una hipótesis nula», Ciencias, no. 42, abril-junio, p. 5.

87 Uno de los argumentos en la disputa institucional fue el de señalar, por parte de Ochoterena, que los integrantes del IBUNAM eran todos profesionales de la biología, mientras que los de la DEB eran un conjunto de naturalistas y aficionados, lo que ha sido reforzado incluso por un comentario hecho por el propio Enrique Beltrán. Vease BELTRÁn, E. (1977), Medio siglo de recuerdos de un biólogo mexicano, México, IMERNAR, p. 59. 
Queremos subrayar que respecto a las ideas teóricas de uno y otro, no hubo diferencias dignas de denominarse como inconmensurables. Mantuvieron prácticas institucionales diametralmente opuestas y cultivaron disciplinas distintas, pero hablar de inconmensurabilidad en tales aspectos significa forzar el concepto hacia campos temáticos para los que no fue elaborado ${ }^{88}$.

Entre las ideas sobre evolucionismo preconizadas por Herrera y las opiniones de Ochoterena no hubo ninguna inconmensurabilidad, porque ambos lo hicieron en el marco de un darwinismo con fuertes tendencias neolamarckistas. La posterior recepción del lysenkismo por parte de Ochoterena tampoco significó una gran ruptura con el paradigma lamarckiano ya que puede entenderse como un esfuerzo de profundización del mismo, de un neolamarckismo, quizá el más erróneo de todos. Pero sí significó una gran ruptura con el darwinismo de ese momento, que era el de la síntesis y el saltacionismo simpsoniano.

El lysenkismo, utilizando el modelo de Programas de investigación de Laka$\operatorname{tos}^{89}$, fue un programa de investigación regresivo, e incluso con rasgos altamente degenerativos ${ }^{90}$, pero sostenido políticamente en la ex - URSS como progresivo y hegemónico. Su recepción en México tuvo paralelismos sorprendentes.

El tema abordado configura la necesidad de una investigación más profunda sobre la historia de la ciencia en México, en la primera mitad del siglo XX y de manera más precisa entre 1930 y 1950, para explorar en los ámbitos de un curioso caso de demarcación teórica donde se decide optar por un programa de investigación regresivo, crecientemente no exitoso, enclaustrar y aislar a un centro de investigación, al mismo tiempo que lograr éxito académico y político, en el marco de una prestigiosa Universidad Nacional.

En contraste con lo anterior, hacia los mismos años, en los círculos de la agronomía y de la zootecnia de la Escuela Nacional de Agricultura (Chapingo), no de la biología, la obra de José Luis de la Loma recepciona la teoría sintética al mismo tiempo que analiza y discute el lysenkismo ${ }^{91}$. En una de

88 Ver KUHN, Th. (1971), La estructura de las revoluciones cientificas, México, FCE,p. 168; Ruiz, R.; Ayala, F. J. (1998), El método en las ciencias. Epistemología y darwinismo, México, Fondo de Cultura Económica, pp. 66-68 y PÉREZ RANSANZ, A. R. (1997). «Cambio científico e inconmensurabilidad», en VELASCO GÓMEZ, A., Racionalidad y cambio científico, México, PAIDOS-UNAM, pp. 71-97

89 LAKATOS, I. (1983), La metodología de los programas de investigación científica, Madrid, Alianza, pp. 145-146

90 PÉrez Ransanz, A.R. (2002), Comunicación personal.

91 De LA LomA, J.L. (1963). Genética general y aplicada, México, UTEHA, p. 267-274. La obra tuvo tres ediciones: 1945, 1954 y 1963 y en todas siguió comparando ambas posturas, 
sus obras dedicada a la enseñanza en el nivel superior, denomina a la síntesis evolutiva como teoría ortodoxa y al lysenkismo como teoría heterodoxa, analiza ambas y se muestra abiertamente partidario de la primera. ${ }^{92}$ Muy distante de la posición mantenida por Ochoterena, señala la necesidad de quitarle el peso de la política a una discusión que debe hacerse con base en argumentos científicos. ${ }^{93}$ En forma vigorosa considera que los experimentos de Morgan y en particular la teoría cromosómica no sólo no se opone a la evolución de las especies, como afirmaba Lysenko, sino que la explica. Dice que la teoría puede tener lagunas, pero que en su concepción general es irrefutable ${ }^{94}$. El estudio de estos dos procesos, ocurridos de manera casi simultánea, debe ser objeto de un nuevo análisis, que por ahora solamente queremos dejar apuntado.

\section{REFERENCIAS}

ACAdemia Lenin de Ciencias Agrícolas (1949), La situación de las Ciencias Biológicas, Ediciones en lenguas Extranjeras, Moscú.

Argueta Villamar, A. (2003), La recepción e introducción del darwinismo en Bolivia y México, un análisis comparativo, tesis de doctorado, Facultad de Ciencias, México, UNAM, $250 \mathrm{pp}$.

BABKov, V. (1996), «Vavilov, Nikolaj Ivanovich», en TORT, P. (Dir.) Dictionnaire du darwinisme et de l'évolution, París, PUF, 3 vols.

BABKov, V. (1996), «Darwinisme Russe», en TORT, P. (Dir.) Dictionnaire du darwinisme et de l'évolution, París, PUF, 3 vols.

BACHELARD, G. (1987), La formación del espiritu científico, 14a ed.,México, Siglo XXI.

Barrera, A.; Bassols Batalla, N. y R. Martín del CAMPO, «Prólogo», en Morton, A.G. (1953), La Genética en la URSS, México, Ediciones del Î́ndice, 5-7 pp.

BELTRÁN, E. (1942), «Alfonso L. Herrera: un hombre y una época», Revista de la Sociedad Mexicana de Historia Natural, tomo III, nos. 1-4, diciembre, pp. 201-210.

inclinándose siempre por la escuela de Morgan, pero sin dejar de analizar los resultados de Lysenko.

92 Para De la Loma, el lysenkismo es un intento de «retorno a las ideas de Lamarck, expuestas en forma muy análoga a la empleada por algunos biólogos del siglo XX, defensores del darwinismo puro, especialmente Spencer», DE LA LOMA, J.L. (1963), Genética general y aplicada, p. 271.

93 DE LA Loma, J. L. (1949), «Ideas nueva e ideas viejas sobre la evolución y la herencia», Revista Chapingo, octubre, pp. 39-43.

94 De La Loma, (1963), p. 272. 
BELTRÁN, E. (1945), «Datos y documentos para la historia de las ciencias naturales en México. II Correspondencia de Alfredo Dugés con Alfonso L. Herrera (18881893)», Revista de la Sociedad Mexicana de Historia Natural, tomo VI, nos. 1-2, junio, pp. 99-106.

BELtrÁn, E. (1953), «Hechos salientes de la biología mexicana en el siglo XX», Memoria del Congreso Científico Mexicano, t. VII, p. 453-482.

BELTRÁN, E. (1964), La teoría de la evolución en el siglo XIX y su vigencia actual Morelia, Universidad Michoacana.

Beltrán, E. (1968), «Alfonso L. Herrera (1868-1942). Primera figura de la Biología mexicana», Revista de la Sociedad Mexicana de Historia Natural, tomo XXIX, diciembre, pp. 37-90.

BELTRÁN, E. (1977), Medio siglo de recuerdos de un biólogo mexicano, México, IMERNAR.

Beltrán, E. (1982), Contribución de México a la Biología. Presente, pasado y futuro, México, CNEB-CECSA, 121 pp.

DE LA LOMA, José Luis (1963), Genética general y aplicada, México, UTEHA.

DE LA LoMA, J. L. (1949), «Ideas nueva e ideas viejas sobre la evolución y la herencia», Revista Chapingo, octubre.

DobZhansky, Th. (1937), Genetics and the origin of the Species, Nueva York, Columbia University Press.

El Colegio NACiOnAL (1969), «Trabajos realizados por El Colegio Nacional durante el año de 1949», Memoria de El Colegio Nacional, IV(4).

HERRERA, Alfonso L. (1897b), Recueil des lois de la biologie générale, México, Oficina Tipográfica de la Secretaría de Fomento.

Herrera, Alfonso L. (1904), Nociones de Biología, México, Secretaría de Fomento

Herrera, Alfonso L. (1924a), Botánica, México Editorial Herrero.

Herrera, Alfonso L. (1924b), Zoología, México, Editorial Herrero.

Herrera, Alfonso L. (1924c), Biología y Plasmogenia, México, Editorial Herrero.

HerrerA, Alfonso L. (1925), Mineralogía y Geología, México, Editorial Herrero.

HuXley, J. (1942), Evolution: the Modern Synthesis, Allen and Unwin, Londres (en español: La evolución. Síntesis moderna, Buenos Aires, Losada, 1946).

KuHN, Th. (1971), La estructura de las revoluciones cientificas, México, Fondo de Cultura Económica.

LAKATOS, I. (1983), La metodología de los programas de investigación científica, Madrid, Alianza Editorial, 315 pp.

LECOURT, D. y otros (1974), El «Caso Lysenko» Barcelona, Anagrama, 151 pp.

Ledesma Mateos, I. (1998), El conflicto entre Alfonso L. Herrera e Isaac Ochoterena y la institucionalización de la Biología en México, tesis doctor en Ciencias, Facultad de Ciencias, México, UNAM. 
LEDESMA MATEOS, I. (2000), «Ochoterena: el hombre, la ciencia y las instituciones científicas», en Ochoterena, Isaac, Obra Científica, Obras I, México, El Colegio Nacional.

MARTín DEL CAMPO, R. (1950), en OCHOTERENA, I., Lecciones de Biología.

MAYR, E. (1942), Systematics and the Origin of the Species, Nueva York, Columbia University Press.

MAYr, E. (1992), Una larga controversia. Darwin y el darwinismo, Barcelona, Crítica.

Medawar, P. B. y Medawar, J. S., (1988), De Aristóteles a Zoológicos, México, Fondo de Cultura Económica.

MeléndeZ, José Luis (1998), Isaac Ochoterena, 1885-1950, Puebla, Secretaría de Cultura, Gobierno del Estado de Puebla.

MONIER, Francoise (2000), «Les disciples de maître Vavilov», L'Express International, no. 2542, 23-29 mars, pp. 20-21.

Moreno De los ARcos, R. (1984), Las polémicas del darwinismo en México, México, UNAM.

Morton, A. G. (1953), La Genética en la U.R.S.S. (trad. de A. Barrera, N. Bassols y R. Martín del Campo), México, Ediciones de Índice, 182 pp.

Ocaranza, F., M. Moctezuma y Morones, S. (1929), Programa de Trabajo del Instituto de Biología, Archivo del Instituto de Biología, CESU, UNAM.

OChoterenA, I. (1916a), «Principios de la teoría de la mutación» de Hugo de Vries (traducción), Boletín de la DEB, t. I, pp. 286-296.

OChoterena, I. (1916b), «Comentarios a la teoría de la mutación», Boletín de la DEB, t. I, pp. 295-301.

OCHOTERENA, I. (1917), «Algunas ideas fundamentales de la obra de Lamarck», Boletín de la $D E B$, t. II, pp. 180-183.

Ochoterena, I. (1922), Lecciones de Biología, México, Secretaría de Educación Pública, Tratado Elemental de Biología, 2a y $3^{\mathrm{a}}$. Ediciones, Imprenta Murguía, (1929) y (1936), respectivamente; $4^{\mathrm{a}}$. Ed. Imprenta Universitaria, UNAM, (1937); $5^{\mathrm{a}}$., $6^{\mathrm{a}}$., $7^{\mathrm{a}}$. y 8. Ediciones, Ediciones Botas, México, (1940), (1941), (1944), (1946), respectivamente; 9a . edición, Ediciones Botas, México, (1950), 428 pp. (póstuma). 11ª . Ed., Editorial Botas, (1960); 13a . Ed., Editorial Botas, (1970), 493 pp.

Ochoterena, I. (2000), Obra Científica, Obras I, (selección y comentarios de Ismael Ledesma Mateos y Antonio Lazcano-Araujo). México, El Colegio Nacional.

PÉREZ RANSANZ, A. R. (1997), «Cambio científico e inconmensurabilidad», en VelASCO GóMEZ, A., Racionalidad y cambio científico, México, PAIDOS-UNAM.

PIÑERO, D. (1996), «La teoría de la evolución en la biología mexicana: una hipótesis nula»,Ciencias, no. 42, abril-junio, 4-8 pp.

Rostand, J. (1971), Ciencia falsa y falsas ciencias, Barcelona, Salvat-Alianza.

RouAIX, P. (1942), «El profesor Alfonso L. Herrera y su labor en la Comisión de Parasitología Agrícola», Rev. de la Soc. Mex. de Hist. Nat., 4, pp. 193-199.

RUIZ GuTIÉRrez, R. (1987), Positivismo y evolución: Introducción del darwinismo en México, México, UNAM. 
Ruiz, R. y F. J. Ayala (1998), El método en las ciencias. Epistemología y darwinismo, México, Fondo de Cultura Económica.

SARKAR, S. (1999), «From the Reaktionsnorm to the adaptive norm: The norm of reaction, 1909-1960», Biology and Philosophy, 14: 235 - 252.

Schmalhausen, I. I. (1949), «Discurso del Académico I. I. Schmalhausen», en Actas de la Academia Lenin de Ciencias Agrícolas de la URSS. La situación de las ciencias biológicas, Editorial en Lenguas Extranjeras, Moscú, pp. 458-464.

Simpson, G. G. (1944), Tempo and Mode of Evolution, Nueva York,Columbia University Press.

SuÁrez y López GuAzo, L. (1999), Determinismo biológico y eugenesia en México, tesis de doctorado, Facultad de Ciencias, , México, UNAM.

VIR (1975), The Institute of Plant Industry, Leningrad, URSS.

VAVILOV, I. (1931), «Mexico and Central America as the principal centre of origin of cultivated plants of the New World», Bulletin of Applied Botany and Plant Breeding, XXVI vol., pp. 179-199

VAVILOV, N. (1951), Estudios sobre el origen de las plantas cultivadas, Buenos Aires, ACME (trad. Felipe Freier, del original ruso-inglés publicado en Bulletin of Applied Botany and Plant Breeding, XVI (2), 1926).

VAVILOV, N. (1951), The Origin, Variation, Inmunity and Breeding of Cultivated Plants. Waltham, Mass. J., Chronica Botanica.

VAVILOV, N. (1992), Origin and geography of cultivated plants. Cambridge, Cambridge University Press. 